\title{
Investigation of photoluminescence and photocurrent in InGaAsP/InP strained multiple quantum well heterostructures
}

\author{
O. Y. Raisky, W. B. Wang, and R. R. Alfano \\ New York State Center of Advanced Technology for Ultrafast Photonic Materials and Application, \\ Department of Physics, The City College and The Graduate School of the City University of New York, \\ Convent Ave.@i38th Street. New York.New York 1003I \\ C. L. Reynolds, Jr. and V. Swaminathan ${ }^{\text {a) }}$ \\ Lucent Technologies, Bell Laboratories, 9999 Hamilton Blvd., Breinigsville, Pennsylvania 1803I
}

(Received 13 August 1996; accepted for publication 30 September 1996)

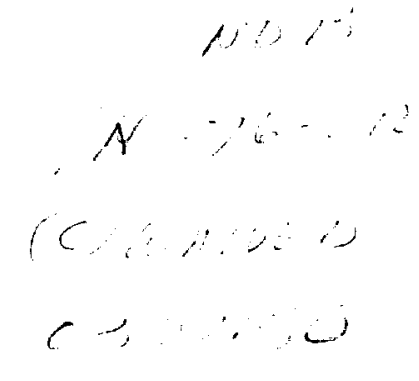

\begin{abstract}
Multiple quantum well InGaAsP/InP $p-i-n$ laser heterostructures with different barrier thicknesses have been investigated using photoluminescence (PL) and photocurrent $(\mathrm{PC})$ measurements. The observed PL spectrum and peak positions are in good agreement with those obtained from transfer matrix calculations. Comparing the measured quantum well PC with calculated carrier escape rates, the photocurrent changes are found to be governed by the temperature dependence of the electron escape time. (1) 1997 American Institute of Physics. [S0021-8979(97)07201-0]
\end{abstract}

\section{INTRODUCTION}

In recent years multiple quantum well (MQW) InGaAsP/ InP heterostructure systems have been an intense reserach area due to their suitability for long wavelength devices, ${ }^{1,2}$ such as infrared detectors, laser, and electro-optical modulators. High radiation resistance of InP-based solar cells makes them potential candidates for space applications. ${ }^{3}$ Several groups have already reported on InGaAs/InP-based ${ }^{4.5} \mathrm{MQW}$ solar cells. The performance of MQW devices strongly depends on recombination and escape processes in the quantum wells (QW). A hetter understanding of these processes and their relationship with structural and compositional parameters of heterostructures is important for improving and developing MQW devices. Photoluminescence (PL) and photocurrent (PC) measurements are reliable sources of information about the carrier escape mechanism and underlying physical processes. ${ }^{6-8}$

In this article, we present our studies on the photoluminescent and photoconductive properties of the MQW InGaAsP/InP $p-i-n$ heterostructures. An efficient escape and collection of photoexcited carriers and factors which influence them are the main concern of this work, because of their importance for photovoltaic applications. The temperature dependence of PL, PC, and dark current (DC) is used to relate the amplitude of photocurrent with the carrier escape rates and the losses due to nonradiative recombination.

\section{SAMPLES}

Two InGaAsP/InP MQW samples with a fixed well width and different barrier thicknesses were grown on $n^{*}$-type $\ln \mathrm{P}$ substrates in a low-pressure (60 Torr) metalorganic chemical vapor deposition (MOCVD) reactor. ${ }^{9}$ The unintentionally doped (light $p$-type) active MQW region consisted of $9 \times 70$ A compressively strained wells of InGaAsP (band gap $\lambda \cong 1.35 \mu$ ) and $8 \times 80$ and $8 \times 150 \mathrm{~A}$ lattice-matched barriers of InGaAsP (band gap $\lambda \cong 1.12 \mu$

\footnotetext{
"Present address: Lucent Technologies, Bell Laboratories, North Andover,
} Massachusetts 01845 samples 382 and 381, correspondingly). High resolution $\mathrm{x}$-ray analysis revealed the well strain to be $\approx 1.5 \%$. The MQW region was confined by $500 \mathrm{~A}$ undoped InGaAsP (band gap $\lambda \cong 1.12 \mu$ ) separate confinement layers and embedded between $n$ - and $p-\operatorname{InP}\left(1.5 \times 10^{18} \mathrm{~cm}^{-3}\right)$ cladding layers (see Fig. 1). The $p$-type InP layer was covered with a 600-A-thick InGaAsP cap (band gap $\lambda \cong 1.12 \mu$ ). For $I-V$ measurements contacts on both MQW samples were formed by evaporating gold at $10^{-6}$ Torr to make ringlike contacts on the top (inner radius $=2 \mathrm{~mm}$ ) and circlelike contacts on the bottom.

\section{METHOD}

A mode-locked $\mathrm{Nd}^{3+}$ :yttrium-aluminum-garnet (YAG) laser with second harmonic generation system $(532 \mathrm{~nm}$ wavelength) was used as an excitation source in all PL measurements, and PL spectra were recorded on $0.25 \mathrm{~m}$ SPEX spectrometer using a standard phase lock-in technique. For low temperature measurements the samples were wired and placed into a temperature-variable cryostat. The $I-V$ and spectra response characteristics were measured on a Keithley 236 Source-Measure unit with combination of the quartz tungsten halogen lamp and $0.25 \mathrm{~m}$ monochromator as a light source.

\section{RESULTS}

\section{A. PL measurements}

PL spectra for both InGaAsP MQW samples at $77 \mathrm{~K}$ and zero voltage bias are shown in Fig. $2\left(\lambda_{e x c}=532 \mathrm{~nm}\right)$. The peak at $1.23-1.24 \mu$ is assigned to the radiative recombination of carriers in the QW (a transition between electron and heavy hole ground states), while the $1.07 \mu$ peak, to which we will later refer as "barrier" peak, is due to the emission from the barrier, cap, and confinement layers. The relative strength of the QW peak is higher for the $150 \mathrm{~A}$ barrier sample than for the $80 \mathrm{~A}$ barrier sample. This observation is attributed to stronger carrier confinement and lower escape rate for the first sample. For both samples the observed full 


\begin{tabular}{|c|}
\hline InGaAsP cap, 600A, $1.12 \mu$ band gap \\
p+ type InP, $0.6 \mu$ \\
InGaAsP, 500A, $1.12 \mu$ band gap \\
MQW active layer \\
well: 70 A InGaAsP, 1.35 $\mu$ band gap \\
barrier: 80/150 A InGaAsP, 1.12 $\mu$ band gap \\
\hline InGaAsP, 500A, $1.12 \mu$ band gap \\
\hline$n+$ type InP buffer, $0.75 \mu$ \\
\hline n+ type InP substrate \\
\hline
\end{tabular}

FIG. 1. Schematic diagram of the $\operatorname{lnGaAsP/InP~MQW~structure.~}$

width at half maximum (FWHM) of the QW peaks is within $10-12 \mathrm{meV}$, which indicates good structural and optical quality of the QW.

The absorption for these InGaAsP/InP MQW samples was also measured and compared with their corresponding PL spectra. The QW PL maxima for both samples were found to be shifted up by $8-10 \mathrm{meV}$ in respect to the excitonic absorption peak. This suggests band-to-band transitions as a dominant radiative recombination process.

The temperature dependence of PL spectra for sample 381 (150 A barrier) at zero voltage bias is shown in Fig. 3. As temperature increases, the following changes are observed in the PL spectra. First, the PL peak positions shift to the long wavelength side, consistent with changes in the energy band gap; second, the PL line intensities significantly

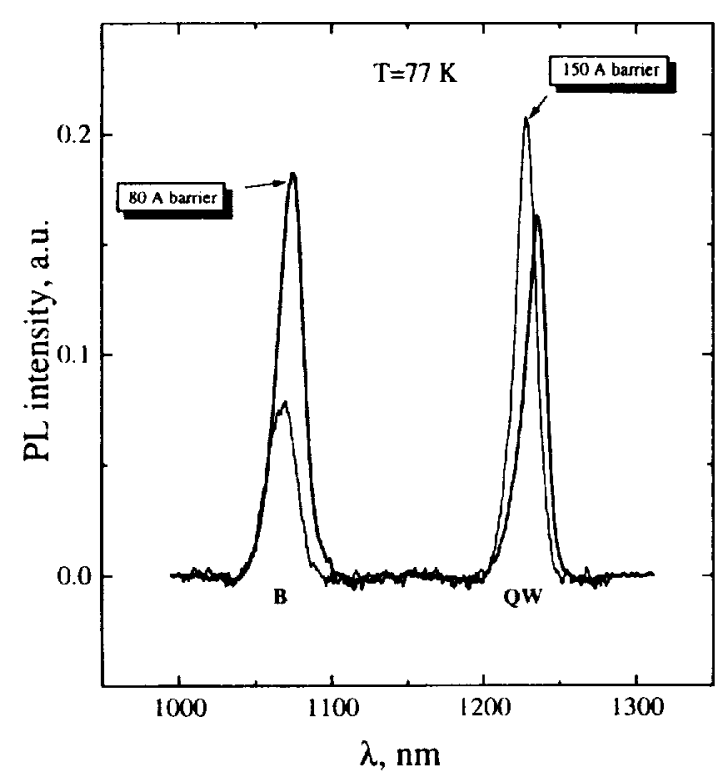

FIG. 2. PL spectra for InGaAsP/InP MQW sample at $77 \mathrm{~K}$.

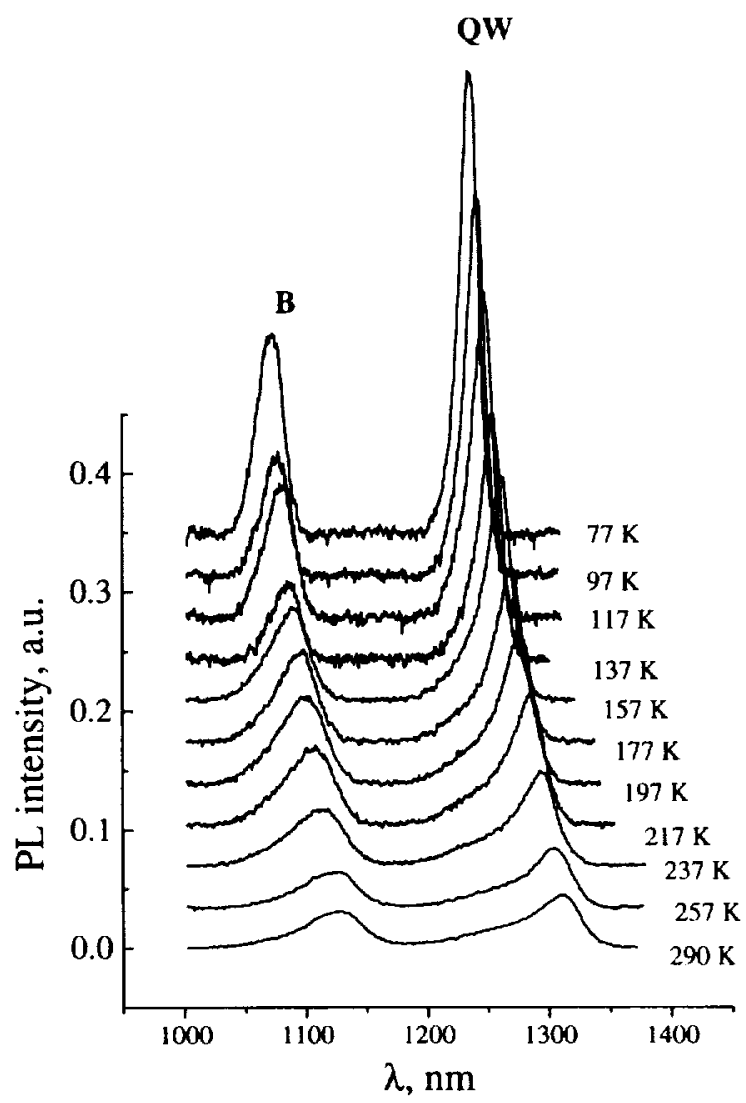

FIG. 3. Evolution of the PL spectrum for MQW InGaAsP/InP with temperature for sample 381 ( $150 \mathrm{~A}$ barrier).

decrease; and third, the PL FWHM broadens. Closer to room temperature, the thermal spreading of the carrier population contributes to an increased radiation from excited QW states as well as to a general broadening of the PL lines.

Figure 4(a) presents the temperature dependence of the QW PL peak position for both samples while the solid lines are the value of the energy band gap for the corresponding bulk InGaAsP alloy calculated using the phenomenological Varshni equation ${ }^{10}$ of $E_{\text {gap }}^{\operatorname{lnGaAP}}(T)=E_{\text {gap }}^{\operatorname{lnGaAsP}}(0)-\alpha \cdot T^{2} /$ $(\beta+T)$, where $T$ is the temperature in $\mathrm{K}, \alpha=6.9 \times 10^{-4}$ $\mathrm{eV} / \mathrm{K}$, and $\beta=327 \mathrm{~K}$. The experimental data show essentially the same temperature dependence as the band gap of the bulk InGaAsP alloy, which allows us to conclude that the $\mathrm{QW}$ energy subbands remain mainly unaffected by the temperature changes. This behavior is similar to the results reported for InGaAs/InP MQW structures."

The ratio of well-to-barrier PL peak intensities $I_{\mathrm{QW}} / I_{B}$ [shown in Fig. 4(b)] provides information about the interrelationship between carrier capture and escape times $\tau_{\text {cap }}$ and $\tau_{\text {esc }}$. The $I_{\mathrm{QW}} / I_{B}$ ratio can be written ${ }^{12}$ as a function of the $\left(n_{\mathrm{QW}} p_{\mathrm{QW}}\right) /\left(n_{b} p_{b}\right)-I_{\mathrm{QW}} / I_{B} \propto\left(n_{\mathrm{QW}} p_{\mathrm{QW}}\right) /\left(n_{b} p_{b}\right)$, where $n_{\mathrm{QW}}, p_{\mathrm{QW}}, n_{b}$, and $p_{b}$ are electron and hole densities in the QW and barrier, respectively, which, in turn, can be expressed in terms of carried escape and capture times $\tau_{\text {esc }}^{n, p}$ and $\tau_{\text {cap }}^{n, p}:\left(n_{\mathrm{QW}} p_{\mathrm{QW}}\right) /\left(n_{b} P_{b}\right) \propto\left(\tau_{\mathrm{exc}}^{n} \tau_{\mathrm{esc}}^{p}\right) /\left(\tau_{\text {cap }}^{n} \tau_{\text {cap }}^{p}\right)$, where $n$ and $p$ indices stand for electron and hole, correspondingly. Taking the ratio of intensities instead of just $l_{\mathrm{OW}}$ or $I_{B}$ alone also reduces the effect of temperature dependence of radiative 


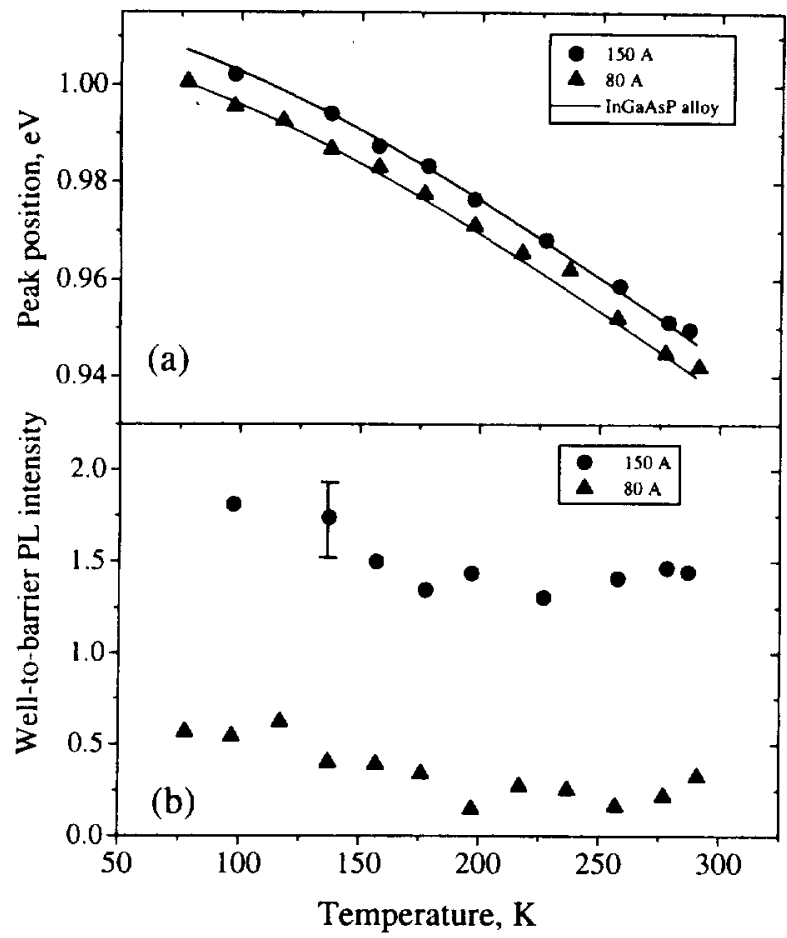

FIG. 4. (a) Quantum well PL line position vs $T$ for InGaAsP/InP MQW. Solid lines-calculated values for band gap of InGaAsP bulk alloy. (b) the ratio of well-to-barrier PL peak intensities vs $T$. Triangles- $-382180 \mathrm{~A}$ barrier), circles-381 (150 A barrier).

recombination rate on analysis ${ }^{12}$ as well as eliminates errors due to pump power fluctuations. As is shown in Fig. 4(b), the ratio predictably reduces with rising temperature as more and more carriers are thermally activated out of the QW, thus effectively decreasing the QW PL. This also can be interpreted as a reduction of the carrier escape time over capture time.

The PL intensities of barrier and QW peaks are displayed in Fig. 5 at different bias voltage. When negative bias

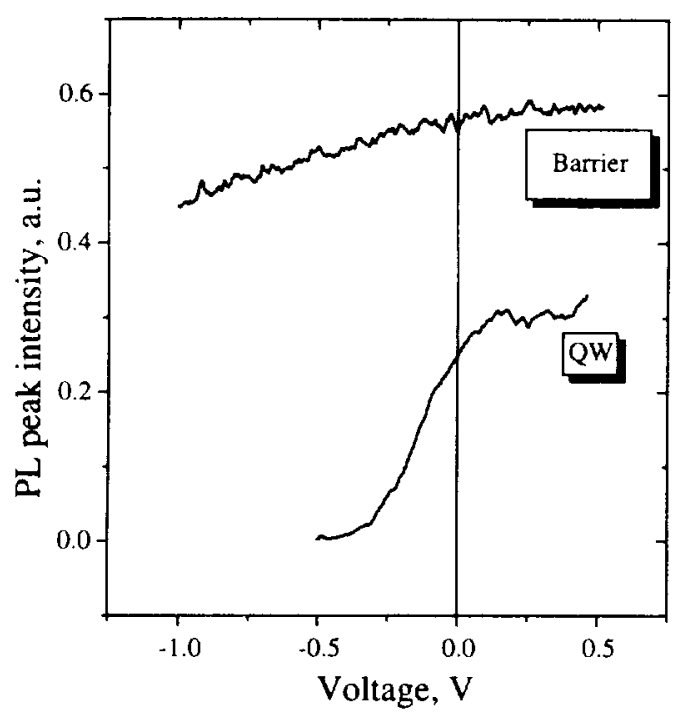

FIG. 5. PL intensity vs applied voltage for InGaAsP/InP MQW at room temperature; sample 283 ( 80 A barrier).

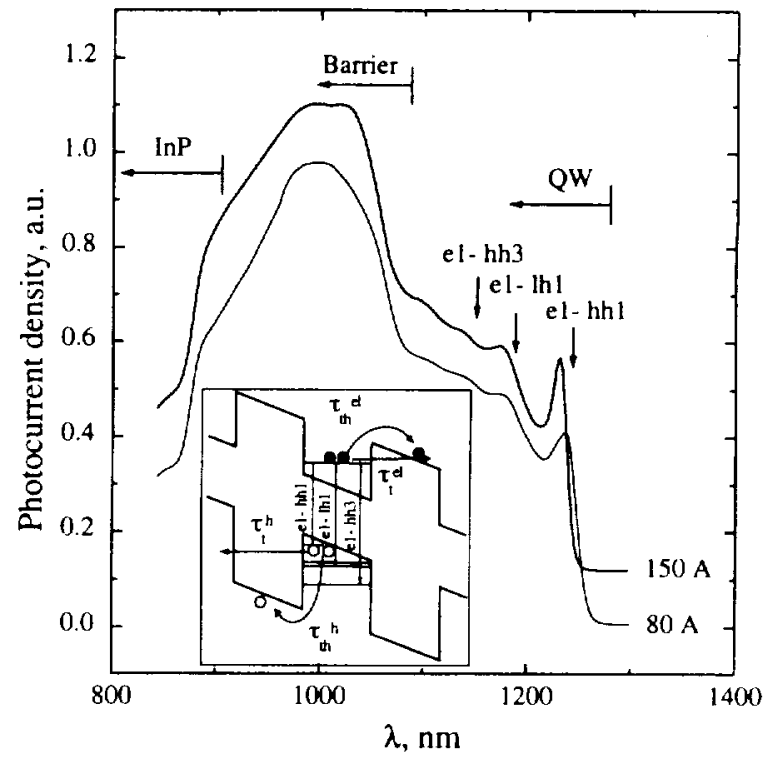

FIG. 6. Spectral response of InGaAsP/InP MQW heterostructure samples at $77 \mathrm{~K}$. The arrows indicate the calculated values of optical transitions in the QW. The inset shows sample's energy dagram with possible escape channels.

is applied, the separation of electron-hole pairs generated inside the undoped MQW region increases, such that the excited carriers can escape before they recombine in the QW. The highly doped layers remain relatively unaffected by the bias and should not exhibit any significant changes in the PL. It can be seen that the QW emission is very sensitive to bias, whereas the barrier PL intensity is almost unaffected. This suggests that most of the barrier PL emission comes from the topmost InGaAsP cap and confinement layer rather than the barriers.

For high pump intensities the photogenerated carriers in the MQW region can effectively screen the built-in field and thus cause change in the measured spectra. To account for this effect, a series of PL spectra at different excitation intensities were measured at room temperature. Over $2.5 \mathrm{de}-$ cades of the pump intensities $\left(0.3-45 \mathrm{~W} / \mathrm{cm}^{2}\right)$. no noticeable line shape or position change was observed. indicating that the excited carrier densities were relatively low and did not affect the internal potential distribution.

\section{B. Photocurrent measurements}

The measured photocurrent density versus irradiative wavelength $J_{\text {photu }}(\lambda)$ for the MQW InGaAsP/InP samples at $77 \mathrm{~K}$ and zero bias is displayed in Fig. 6. The curves were normalized and corrected for the system spectral response. It can be seen that, compared to the InP part. the QW and barrier regions make a significant contribution to the total current, which has been observed throughout the entire investigated temperature range. The difference between the 150 and $80 \mathrm{~A}$ samples is shown in Fig. 6. The sample with 150 A barriers produces sharper $e l \rightarrow h h l$ and $e l-l h l$ QW peaks and more pronounced barrier line, which is consistent with thicker barriers in this sample. 


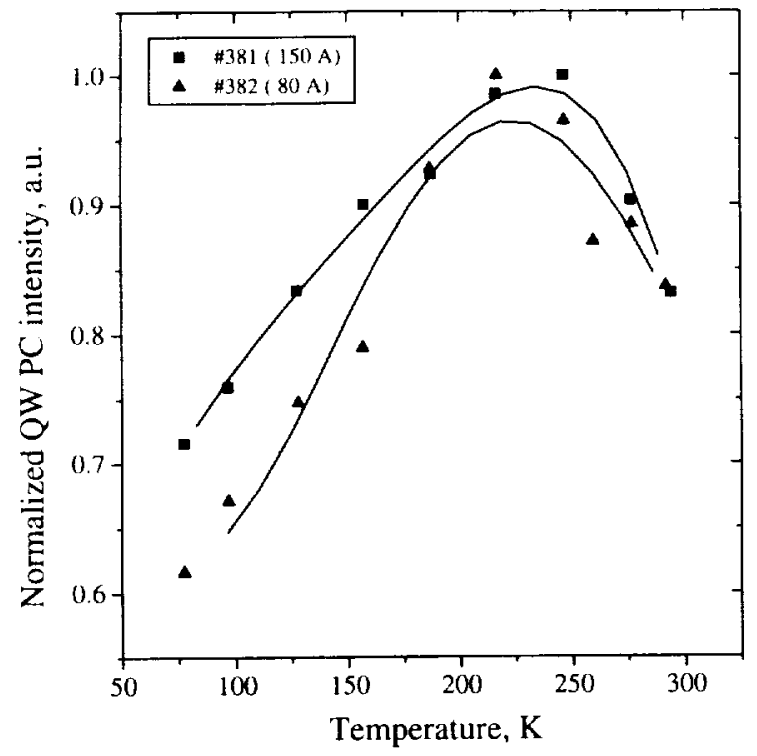

FIG. 7. Integrated $Q W$ photocurrent vs $T$ for InGaAsP/InP MQW samples Squares-381 (150 A barrier), triangles-382 (80 A barrier). Solid line are drawn to guide the view.

Figure 7 shows normalized integrated QW photocurrent density $J_{\text {photo }}(\lambda)$ versus temperature for the 150 and $80 \mathrm{~A}$ barrier samples. Both curves show similar behavior with an initial rise to a maximum near $\approx 220 \mathrm{~K}$ and a decline towards room temperature. The rise portion can be attributed to the thermal activation of photoexcited carriers in the QW; the decline portion is most likely due to some thermo-activated non-radiative process.

\section{Dark current measurements}

Figure 8 shows the dark current (DC) density curves $J_{\mathrm{DC}}(V)$ measured at room temperature. The differences between samples are most noticeable in the reverse bias portion

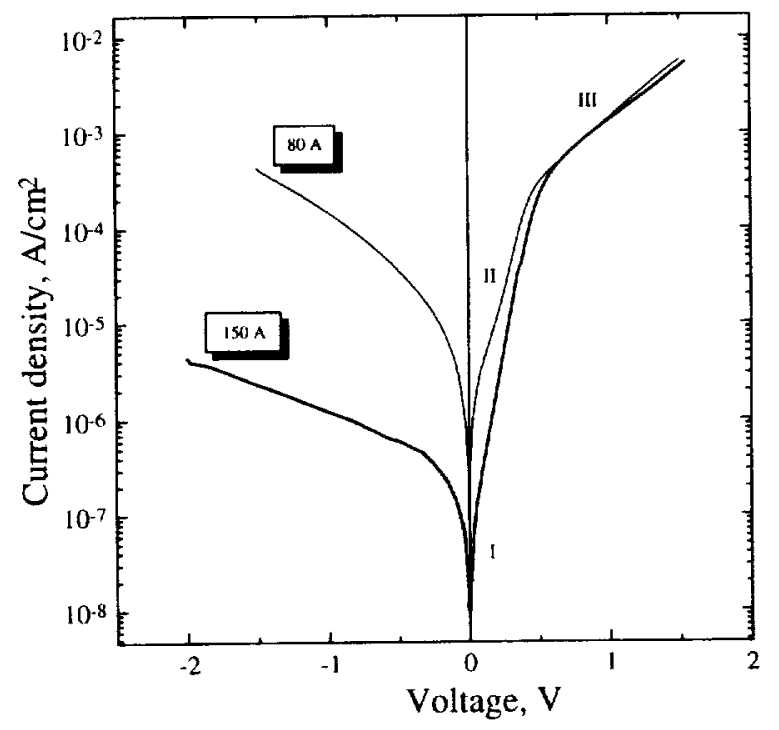

FIG. 8. Dark current density for the InGaAsP/InP MQW samples at room temperature.

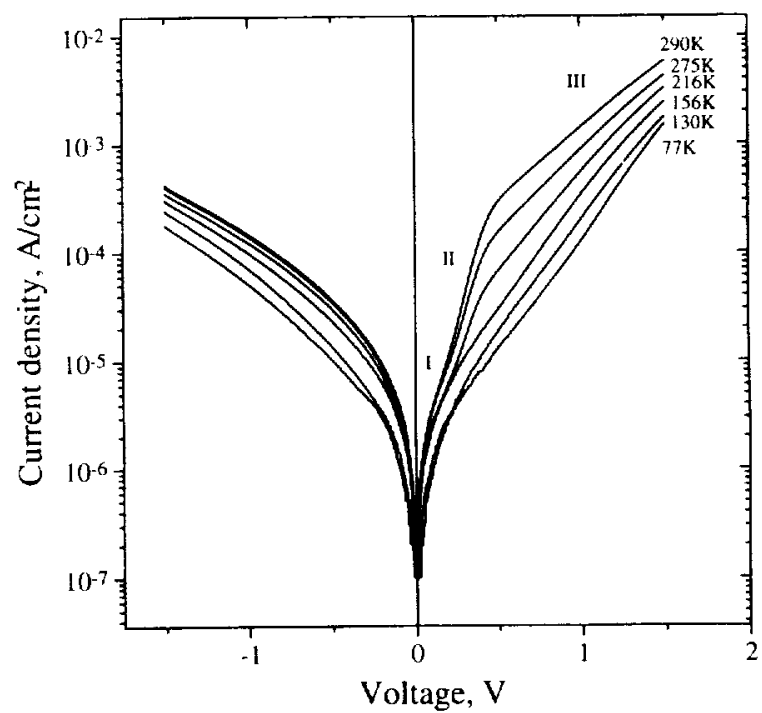

FIG. 9. Temperature dependence of dark current density for InGaAsP/InP MQW. Sample $382(80 \mathrm{~A}$ barrier $)$.

of the $I-V$ characteristics, where variations in internal built-in tields are enhanced by the external voltage. For a given negative bias, a larger dark current is observed for more narrow barrier.

In the forward bias domain, three components are distinguished, denoted by I, II, and III in Fig. 8, which are common to both samples. The relative strength of each component depends differently on the temperature, as seen in Fig. 9. The second component (II) begins to appear for $T>180-$ $210 \mathrm{~K}$ and completely vanishes below these temperatures for the 382 sample ( $80 \mathrm{~A}$ barrier). Similar behavior was observed in the other sample. This fact is compared with the observed PC decrease for $T>200 \mathrm{~K}$ in Fig. 7, and it is concluded that these trends in PC and DC are related to each other and probably caused by the same thermally activated process. The value of the diode ideality factor for the second DC component is estimated to be $\approx 2$, which indicates that this component is due to the contribution from the recombination-generation current. Therefore, we believe that the reduction of PC is caused by the thermo-activated nonradiative recombination on impurity levels.

\section{MODEL}

A model is assumed in which the photoexcited carriers successfully escaping from quantum wells contribute to the QW part of photocurrent. The photocurrent density $J_{\text {phot }}$ can be then written as ${ }^{13} J_{\text {phoro }} \cong q n_{2 D} 1 / \tau^{\text {esc }}$, where $q$ is the electron charge, $n_{2 D}$ the $2 D$ density of the photogenerated carriers, and $1 / \tau^{\mathrm{esc}}$ the escape rate. The $n_{2 D}$ is assumed to be a relatively weak function of the external factors which determine the carrier escape rate, such as temperature and electric field. Therefore the temperature dependence of the QW PC should be mainly controlled by the corresponding changes in the escape rate.

The temperature dependence of carrier escape rates is evaluated for electrons and holes. Figure 10 presents the cal- 


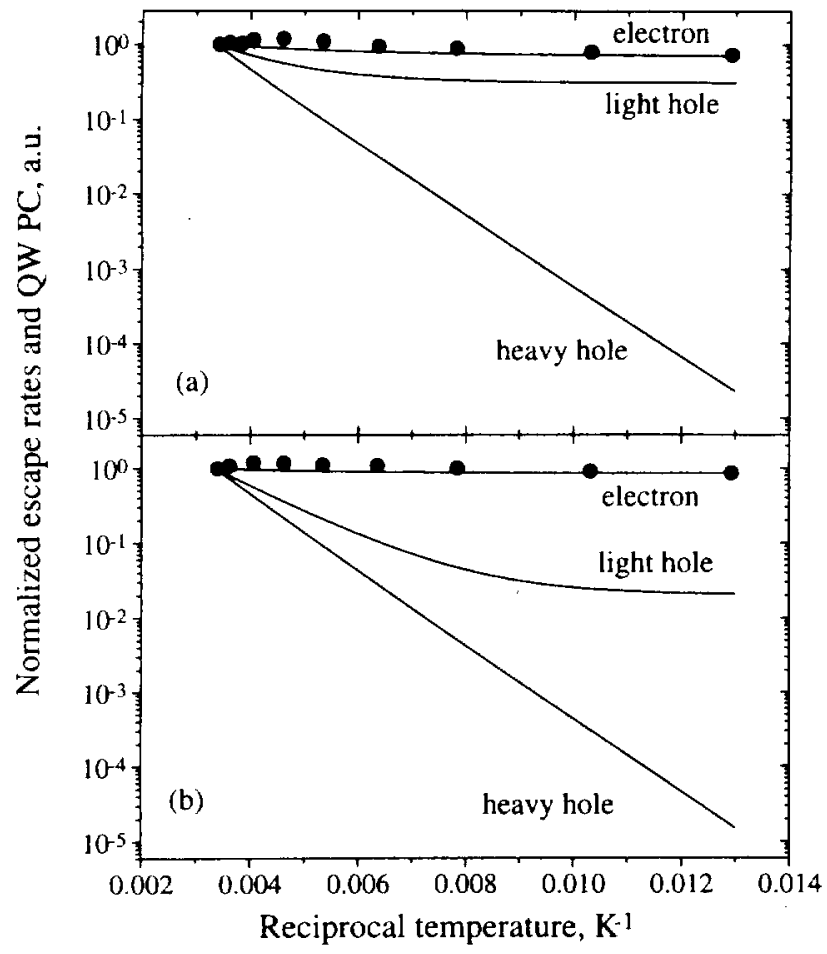

FIG. 10. Calculated escape rate $1 / \tau$ (solid lines) and experimental photocurrent PC (circles). All the curves are normalized to their corresponding values at room temperature. (a) sample $381(150 \mathrm{~A}$ barrier). (b) $382(80 \mathrm{~A}$ barrier).

culated escape rates and the measured QW PC versus reciprocal temperature. To make the comparison easier all the curve were normalized to their corresponding values at room temperature. In modeling, escape rates arising from both tunneling and thermionic emission are considered, and it is assumed that these processes were independent of each other. The inset in Fig. 6 schematically shows the two escape channels for the photoexcited carriers in the QW.

The total escape time $\tau_{\text {total }}$ can be written as (esc index in $\tau$ is omitted for the brevity)

$$
\frac{1}{\tau_{\text {total }}}=\frac{1}{\tau_{t}}+\frac{1}{\tau_{\mathrm{th}}},
$$

where $\tau_{\text {th }}$ and $\tau_{t}$ are thermionic emission and tunneling times in the quantum well, respectively. The $\tau_{\text {th }}$ is given by ${ }^{14}$

$$
\frac{1}{\tau_{\mathrm{th}}}=\frac{1}{w} \cdot \sqrt{\frac{k T}{2 \pi m_{w}}} \exp \left\{-\frac{E_{\mathrm{bar}}(F)}{k T}\right\},
$$

where $m_{w}$ is the effective carrier mass in the quantum well, $w$ the well width, and $E_{\text {barr }}(F)$ the barrier height for $n$-th energy subband. In the presence of an electric field $F$, the barrier height can be written as

$$
E_{\text {barr }}(F)=\Delta E_{c, \nu}-E_{n}-q \cdot \frac{F w}{2},
$$

where $\Delta E_{c, \nu}$ is a conduction or valence band offset, $E_{n}$ the energy of $n$-th energy subband measured from the center of the well, and $q$ the electron charge.
The tunneling escape time $\tau_{t}$ for an arbitrary potential $V(z)$ can be written as ${ }^{15}$

$$
\begin{aligned}
\frac{1}{\tau_{t}}= & \frac{1}{w^{2}} \cdot \frac{\pi n \hbar}{2 m_{w}} \\
& \times \exp \left\{-\frac{2}{\hbar} \int_{0}^{b} \sqrt{2 m_{b}\left(q V(z)-E_{n}-q F z\right)} d z\right\},
\end{aligned}
$$

where $b$ is the barrier width and $m_{b}$ is the effective carrier mass in the barrier.

\section{DISCUSSION}

To calculate the carrier escape rates, the confined energy states for electrons and holes were found using the standard transfer matrix technique. ${ }^{16}$ In our calculations it is assumed that $\Delta E_{V}=0.61 \Delta E_{\text {gap }}$, where $\Delta E_{V}$ is a valence band offset for InGaAsP/InP systems and $\Delta E_{\text {gap }}$ the total band gap discontinuity. The estimated $\Delta E_{V}$ and $\Delta E_{C}$ were 112 and 72 $\mathrm{meV}$, correspondingly. Other material parameters were obtained as a linear interpolation between those of $\operatorname{InP}$ and $\mathrm{In}_{0.53} \mathrm{Ga}_{0.47} \mathrm{As} .{ }^{17}$ The calculated values of the $\mathrm{QW}$ optical transitions are denoted by arrows in Fig.6. The theoretical peak positions tend to fall behind the experimental photoresponse peaks on the energy scale, which is most likely due to the overestimate of the carrier effective masses obtained from interpolation. The calculations showed that the effect of different barrier thickness on peak positions was very small (less than $1 \mathrm{meV}$ shift) compared to the uncertainties in material parameters.

Another important issue in calculating escape rates is the adequate assessment of internal built-in fields. Background doping of the MQW region results in a variation of the built-in electric field, and thus the calculation of energy states in a $p-i-n$ structure requires some knowledge about the distribution of this field. Figure 11 presents the calculated field distribution in the MQW region using the method described by Lundstrom and Schuelke. ${ }^{18}$ In the transfer matrix calculations the inhomogeneous field inside the MQW region is replaced with some constant field, obtained by averaging across the MQW region. The resulting error in energy subbands was estimated to be less than $5 \%$.

The calculated temperature dependence of the carrier escape rate $1 / \tau_{\text {total }}(T)$ has been compared with the measured QW photocurrent density $J_{\text {photo }}(T)$ in Fig. 10. It is found that the change of the measured photocurrent with temperature mostly followed the changes of the electron escape rate. This is expected, since in InGaAsP/InP QWs, electrons are the carriers with the lowest escape time. The activation energy obtained from single exponential fitting to the $J_{\text {photo }}$ $\propto f(1 / T)$ plot gives a value of $33 \pm 2 \mathrm{meV}$ for the effective barrier height, which tends to be somewhat more than our calculated value of $25 \mathrm{meV}$. Since effective barrier height is directly proportional to the electric field, this discrepancy may be a result of the averaging procedure we attempted earlier. In order to explain the reduction of $J_{\text {photo }}(T)$ for $T>200 \mathrm{~K}$, our model should be expanded and we should take into account various nonradiative recombination processes, which is out of the scope of this work. As mentioned 


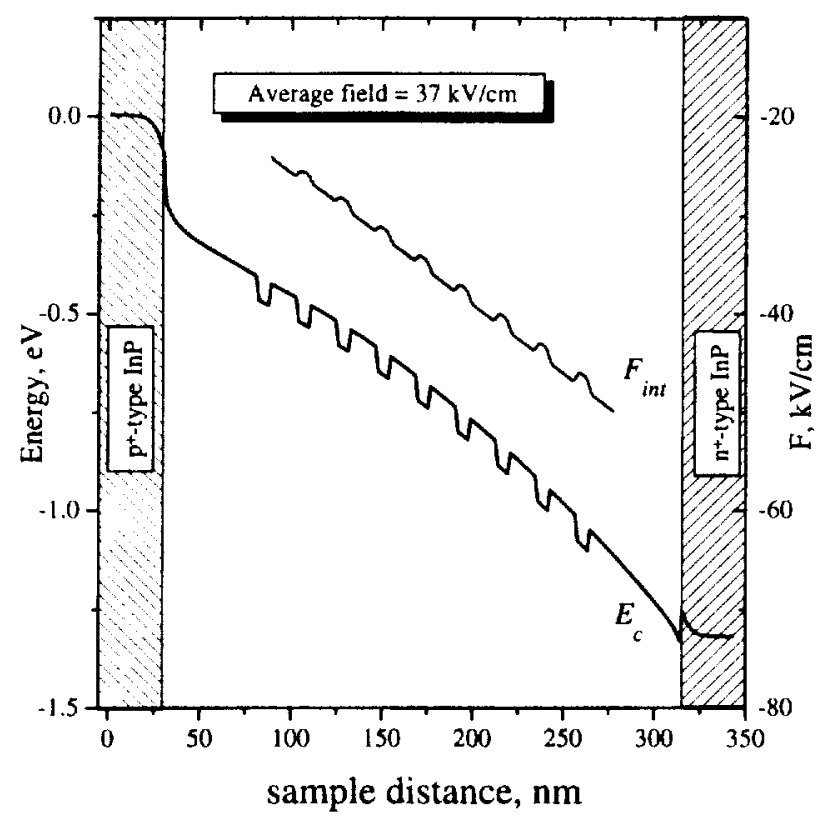

FIG. 11. The calculated equilibrium energy band diagram and internal electric field distribution for the InGaAsP/InP MQW sample 381 (150 A barrier). $E_{c}$-conduction band edge; $F_{\text {int }}$-internal built-in field.

earlier, this reduction of $J_{\text {photo }}(T)$ may occur when the photogenerated carriers get trapped or recombine on impurity levels located inside the energy band gap, which effectively diminishes or eliminates their contribution to the total PC.

From this investigation of InGaAsP/InP based MQW heterostructures, information was learned which can be important for designing efficient MQW devices, such as photodiodes and solar cells. This study showed that lowering temperature below $200 \mathrm{~K}$ had an overall degrading effect on the photocurrent in InGaAsP/InP based MQW; however, in the range of 200-250 K a small rise of PC magnitude was observed which was attributed to the reduction of losses due to the carrier recombination on impurity levels. The difference in barrier thickness-80 A vs $150 \mathrm{~A}$-has the most profound effect on amplitude of PL and PC. The sample with $150 \mathrm{~A}$ barriers had $\approx 1.8$ more intense QW PL and twice as less PC under the same experimental conditions. Because barrier thickness mostly affects tunneling, this means that tunneling through the barriers is a crucial element in carrier escape for this particular range of thicknesses $(80-150 \mathrm{~A})$. The electrons appeared to play a more significant role in defining the photoconductive properties in these InGaAsP/InP hetero- structures than their hole counterpart, as was discussed above in the case of escape rates. The transfer matrix method and interpolation scheme used to calculate energy subbands in the InGaAsP QW show good agreement with the experiment.

In conclusion, MQW InGaAsP/InP $p$ - $-i-n$ heterostructures with different barrier thicknesses have been investigated by measuring PL, PC, and DC. The observed PL and spectral line positions were found to be in good agreement with those obtained from transfer matrix calculations. Comparing the measured QW PC with calculated carrier escape rates, the photocurrent changes were found to be governed by the temperature dependence of the electron escape time. An activation energy obtained from the PC data seems to support this conclusion.

\section{ACKNOWLEDGMENT}

This research at CCNY is supported in part by NASA research Grant No. NAG 3-1490 and the New York State Technology Foundation.

'B. F. Levine, J. Appl. Phys. 74, R1 (1993).

${ }^{2}$ B. W. Takasaki, J. S. Preston, J. D. Evans, and J. G. Simmons, Can. J. Phys. 70, 1017 (1992).

${ }^{3}$ D. J. Flood and 1. Weinberg, Optoelectron., Devices Technol, 9, 451 (1994).

${ }^{4}$ N. G. Anderson and S. J. Wojtczuk, J. Appl. Phys. 79, 1973 (I996).

${ }^{5}$ A. Freundilch, V. Rossignol, M. F. Viela, P. Renaud, A. Bensaoula, and N. Medleci, Proceedings of the 1994 IEEE World Conference on Photovoltuic Conversion (IEEE, New York, 1995), p. 1886.

${ }^{\circ}$ D. Kinder, R. J. Nicholas, P. N. Stavrinou, S. K. Haywood, L. Hart, M. Hopkinson, J. P. R. David, and G. Hill, Semicond. Sci. Technol. 11, 34 (1996)

${ }^{7}$ E. H. Reihten, A. Person, T. Y. Wang, K. L. Fry, and G. B. Stringfellow, J. Appl. Phys. 66, 5554 (1994).

${ }^{8}$ H. Schneider, J. Wagner, and K. Ploog, Solid-State Electron. 37, 881 (1994).

${ }^{9}$ O. Y. Raisky, W. B. Wang. R. R. Alfano, C. L. Reynolds, Jr., and V. Swaminathan, Proceedings of the 9 th conference on Hot Carriers in Semiconductors (Plenum, New York, 1996), p. 11.

${ }^{10}$ Y. P. Varshni, Physica (Amsterdam) 34, 149 (1967).

${ }^{11}$ H. Temkin, D. Gershoni, and M. B. Panish, in Epitaxial Microssructures edited by A. C. Gossard (Academic, New York, 1994).

${ }^{12}$ S. Marcinkevicius, U. Olin, and G. Treideris, J. Appl. Phys. 74, 3587 (1993).

${ }^{13}$ A. M. Fox. D. A. B. Miller, G. Livesku, J. E. Cunningham, and W. Y. Jan, 27, 2281 (1991).

${ }^{14}$ H. Schneider and K. Von Klitzing, Phys. Rev. B 38, 6160 (1988).

${ }^{15}$ L. D. Landau and E. M. Lifshitz, Quantum Mechanics, Non-Relativistic: Theory, 3rd ed. (Pergamon, New York, 1977), pp. 178-181.

${ }^{16}$ A. Harwit, J. S. Harris, Jr, and A. Kapitulnik. J. Appl. Phys. 60. 1973 (1996).

${ }^{17}$ D. Gershoni, C. H. Henry, and G. A. Baraff, 29. 3211 (1986).

${ }^{1 \times}$ M. S. Lundstrom and R. J. Schuelke, IEEE Trans. Electron Devices ED30. $1151(1983)$. 